\title{
Circulating microbiome in blood of different circulatory compartments
}

Schierwagen, Robert; Alvarez-Silva, Camila; Madsen, Mette Simone Aae; Kolbe, Carl Christian; Meyer, Carsten; Thomas, Daniel; Uschner, Frank Erhard; Magdaleno, Fernando; Jansen, Christian; Pohlmann, Alessandra; Praktiknjo, Michael; Hischebeth, Gunnar T; Molitor, Ernst; Latz, Eicke; Lelouvier, Benjamin; Trebicka, Jonel; Arumugam, Manimozhiyan Published in:

Gut

DOI:

10.1136/gutjnl-2018-316227

Publication date:

2019

Document version

Peer reviewed version

Document license:

CC BY-NC

Citation for published version (APA):

Schierwagen, R., Alvarez-Silva, C., Madsen, M. S. A., Kolbe, C. C., Meyer, C., Thomas, D., Uschner, F. E., Magdaleno, F., Jansen, C., Pohlmann, A., Praktiknjo, M., Hischebeth, G. T., Molitor, E., Latz, E., Lelouvier, B., Trebicka, J., \& Arumugam, M. (2019). Circulating microbiome in blood of different circulatory compartments. Gut, 68(3), 578-580. https://doi.org/10.1136/gutjnl-2018-316227 
Title:

Circulating microbiome in blood of different circulatory compartments.

\section{Authors:}

Robert Schierwagen $^{1 *}$, Camila Alvarez-Silva ${ }^{2 *}$, Mette Simone Aae Madsen ${ }^{2}$, Carl Christian Kolbe ${ }^{3}$, Carsten Meyer ${ }^{4}$, Daniel Thomas ${ }^{4}$, Frank Erhard Uschner ${ }^{1}$, Fernando Magdaleno ${ }^{1}$, Christian Jansen ${ }^{1}$, Alessandra Pohlmann'1, Michael Praktiknjo', Gunnar T. Hischebeth ${ }^{5,6}$, Ernst Molitor ${ }^{5,6}$, Eicke Latz ${ }^{3,7,8,9}$, Benjamin Lelouvier ${ }^{10}$, Jonel Trebicka ${ }^{1,11,12,13 \#}$ and Manimozhiyan Arumugam ${ }^{2 \#}$

* share first authorship

\# shared corresponding author

\section{Affiliations:}

${ }^{1}$ Department of Internal Medicine I, University of Bonn, Bonn, Germany

2 The Novo Nordisk Foundation Center for Basic Metabolic Research, Faculty of Health and Medical Sciences, University of Copenhagen, Copenhagen, Denmark

${ }^{3}$ Institute of Innate Immunity, University of Bonn, Bonn, Germany

${ }^{4}$ Department of Radiology, University of Bonn, Bonn, Germany

${ }^{5}$ Institute of Medical Microbiology, Immunology and Parasitology, University of Bonn, Bonn, Germany

${ }^{6}$ German Center of Infection Research (DZIF), Partner site Cologne-Bonn, Germany

${ }^{7}$ Division of Infectious Diseases and Immunology, University of Massachusetts Medical School, Worcester, MA, USA

${ }^{8}$ German Center for Neurodegenerative Diseases (DZNE), Bonn, Germany

${ }^{9}$ Centre for Inflammation Research, Norwegian University of Science and Technology, Trondheim, Norway

${ }^{10}$ Vaiomer, Labège, France

${ }^{11}$ European Foundation for the Study of Chronic Liver Failure, Barcelona, Spain

12 Institute for Bioengineering of Catalonia, Barcelona, Spain

${ }^{13}$ Department of Medical Gastroenterology and Hepatology, Odense University Hospital, Odense, Denmark

Corresponding Authors:

Jonel Trebicka: Department of Internal Medicine I, University of Bonn, Sigmund-Freud-Str. 25, 53127 Bonn. jonel.trebicka@ukbonn.de, Tel: +4922828715507

Manimozhiyan Arumugam: The Novo Nordisk Foundation Center for Basic Metabolic Research, Faculty of Health and Medical Sciences, University of Copenhagen, Blegdamsvej 3B, DK-2200 Copenhagen N. arumugam@sund.ku.dk, Tel: +45 35337581

Word count: 522 
We read with interest the recent review by Tilg et al. ${ }^{1}$, which summarised the role of microbiota in liver diseases and pointed out that a causal link with systemic inflammation has still not been established. This letter fills in this gap and provides an analysis of the circulating microbiota in portal vein as the link between gut and liver. The access to portal circulation is possible during the implantation of a transjugular intrahepatic portosystemic shunt (TIPS). Therefore, we characterized the circulating microbiome in portal vein (first venous outflow in gut-liver-axis), liver outflow, central venous blood, and peripheral venous blood from 7 patients with decompensated liver cirrhosis receiving TIPS for either variceal bleeding $(n=3)$ or refractory ascites $(n=4)$ (mean MELD 8.4 [range 613], CHILD A $n=4$, CHILD B $n=3$ ) (Figure 1A). We performed 16S ribosomal RNA (rRNA) gene sequencing of buffy coat samples and identified 65 genera belonging to 4 phyla (predominantly Proteobacteria) in this cohort (Supplementary Figure 1 and Figure 1B). Blood microbiome phylum compositions identified in our study agreed with previous findings investigating the peripheral blood microbiome in buffy-coat samples from liver fibrosis patients ${ }^{2}$ as well as healthy individuals ${ }^{3}$, but differed from the gut microbiome measured in fecal samples, where Bacteroidetes and Firmicutes are predominant ${ }^{2}$.

Although overall bacterial community structure did not show a compartment-specific clustering as assessed by Bray-Curtis dissimilarity measures (Figure 1C), the abundance of several genera varied strongly in circulation forming compartment-specific patterns (Figure 1D, using DESeq2 for differential abundance analysis ${ }^{4}$ ). To verify whether the bacteria observed in 16S rRNA gene sequencing were viable in blood, we performed aerobic and anaerobic cultivation of blood from the four sites of five additional TIPS patients. Three out of five patients showed positive cultivation with Staphylococcus and one that showed bacterial growth of Acinetobacter, both abundant in the sequencing data (Supplementary Figure 1). These results demonstrate that circulating microbiota are indeed viable.

Bacterial infections are frequent in cirrhotics, often trigger acute-on-chronic liver failure and are associated with high mortality 5 . A number of cytokines, which might show immune dysfunction, systemic inflammation and oxidative stress have been linked to decompensation, acute-on-chronic liver failure and mortality ${ }^{6-10}$.

To elaborate on the relationship with systemic inflammation, we measured cytokine levels in serum of the same patients and compartments except central venous blood. Inflammatory cytokines formed patient-specific clusters (Figure 2A) and their individual measurements showed robust associations with the abundance of blood microbiome genera measured by Spearman correlation (Figure 2B), which establish the association of circulating microbiota with systemic inflammation. 
To the best of our knowledge, this study is the first to track the major part of microbiome of portalvenous blood through liver into central venous blood and circulating into peripheral blood. With this study we provide snapshots of the circulating blood microbiome, its compartment-specific patterns, viability of the microbial members and their association with inflammation. Further studies with larger cohorts are required to better understand the role of circulating microbiome and expand the knowledge about gut microbiome contribution to liver diseases reviewed recently ${ }^{1}$.

Ethical approval: The ethics committee of the University Clinic Bonn in agreement with the Declaration of Helsinki permitted the study (No. 295/16). 


\section{Acknowledgments}

The authors thank Gudrun Hack and Silke Bellinghausen for excellent technical assistance. The authors were supported by grants from the Deutsche Forschungsgemeinschaft (SFB TRR57), Cellex Foundation, Novo Nordisk Foundation (NNF10CC1016515 and NNF16CC0020896), and European Union's Horizon 2020 research and innovation program (No 668031). The funders had no influence on study design, data collection and analysis, decision to publish or preparation of the manuscript.

\section{Author Contributions}

Conceptualization, R.S., C.A.S., J.T. and M.A.; Methodology, R.S., C.A.S., G.T.H., E.M., J.T. and M.A. ; Formal Analysis, R.S., C.A.S., J.T. and M.A.; Investigation, R.S., C.A.S., M.S.A.M., C.C.K., C.M., D.T., F.E.U., F.M., C.J., A.P., M.P., G.T.H.; Resources, C.C.K., C.M., D.T., F.E.U., F.M., C.J., A.P., M.P., G.T.H., E.L.; Data Curation, B.L.; Writing - Original Draft, R.S., C.A.S., E.M., E.L., B.L., J.T. and M.A.; Visualization, R.S., C.A.S., J.T. and M.A.; Supervision, J.T. and M.A.

\section{Declaration of Interests}

B. Lelouvier is an employee of Vaiomer. 


\section{References}

1. Tilg, H., Cani, P. D. \& Mayer, E. A. Gut microbiome and liver diseases. Gut 65, 2035-2044 (2016).

2. Lelouvier, B. et al. Changes in blood microbiota profiles associated with liver fibrosis in obese patients: A pilot analysis. Hepatology 64, 2015-2027 (2016).

3. Païssé, S. et al. Comprehensive description of blood microbiome from healthy donors assessed by $16 \mathrm{~S}$ targeted metagenomic sequencing. Transfusion 56, 1138-1147 (2016).

4. Love, M. I., Huber, W. \& Anders, S. Moderated estimation of fold change and dispersion for RNAseq data with DESeq2. Genome Biol. 15, 550 (2014).

5. Fernández, J. et al. Bacterial and fungal infections in acute-on-chronic liver failure: prevalence, characteristics and impact on prognosis. Gut (2017). doi:10.1136/gutjnl-2017-314240

6. Hackstein, C.-P. et al. Gut microbial translocation corrupts myeloid cell function to control bacterial infection during liver cirrhosis. Gut 66, 507-518 (2017).

7. Clària, J. et al. Systemic inflammation in decompensated cirrhosis: Characterization and role in acute-on-chronic liver failure. Hepatology 64, 1249-1264 (2016).

8. Lehmann, J. M. et al. Circulating CXCL10 in cirrhotic portal hypertension might reflect systemic inflammation and predict ACLF and mortality. Liver Int. (2017). doi:10.1111/liv.13610

9. Jansen, C. et al. Increase in liver stiffness after transjugular intrahepatic portosystemic shunt is associated with inflammation and predicts mortality. Hepatology (2017). doi:10.1002/hep.29612

10. Berres, M.-L. et al. CXCL9 is a prognostic marker in patients with liver cirrhosis receiving transjugular intrahepatic portosystemic shunt. J. Hepatol. 62, 332-339 (2015). 


\section{Figures}

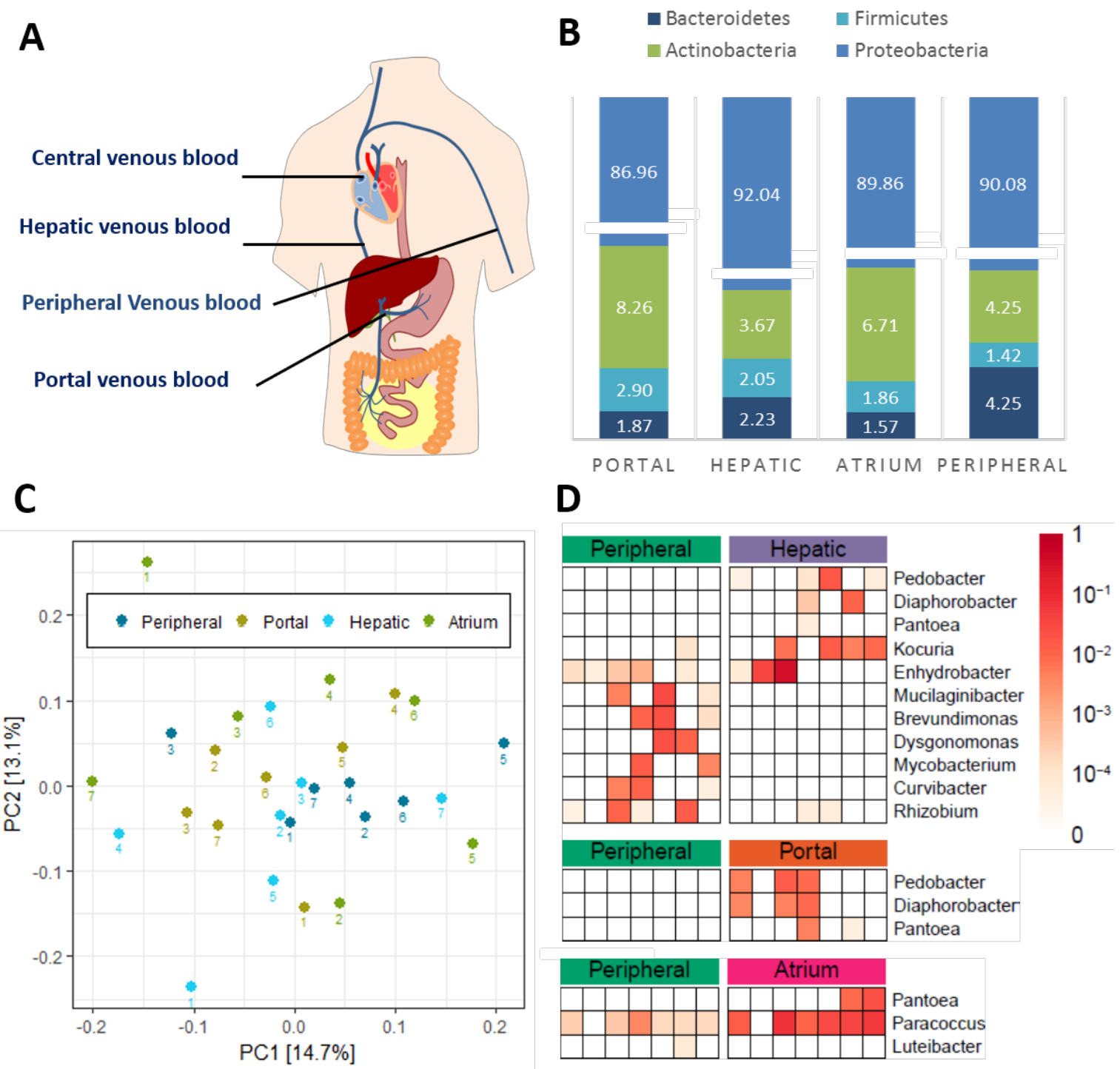

Figure 1. A) During implantation of a transjugular portosystemic shunt (TIPS), central, hepatic and portal venous blood, as well as peripheral blood, was collected from 7 patients. B) Phylum composition of buffy coat samples from different compartments. C) Microbial community compositions did not differ significantly between compartment. D) Differentially abundant genera in the cohort. Portal, hepatic and central (Atrium) venous blood microbiome were compared to that of peripheral blood. 
A

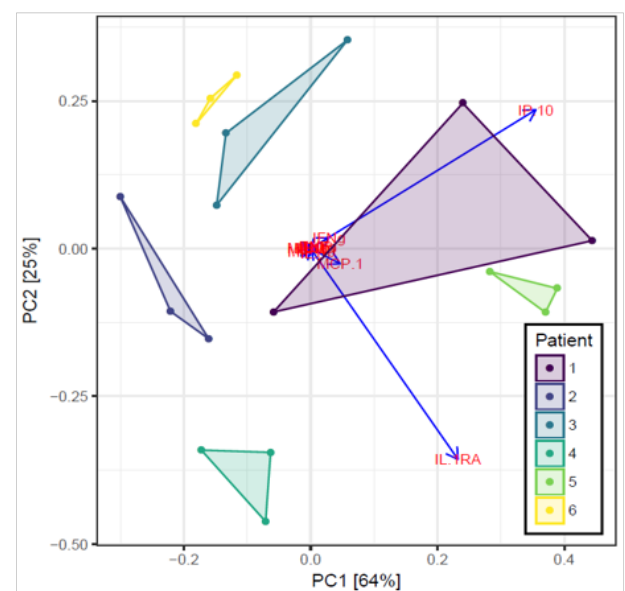

B
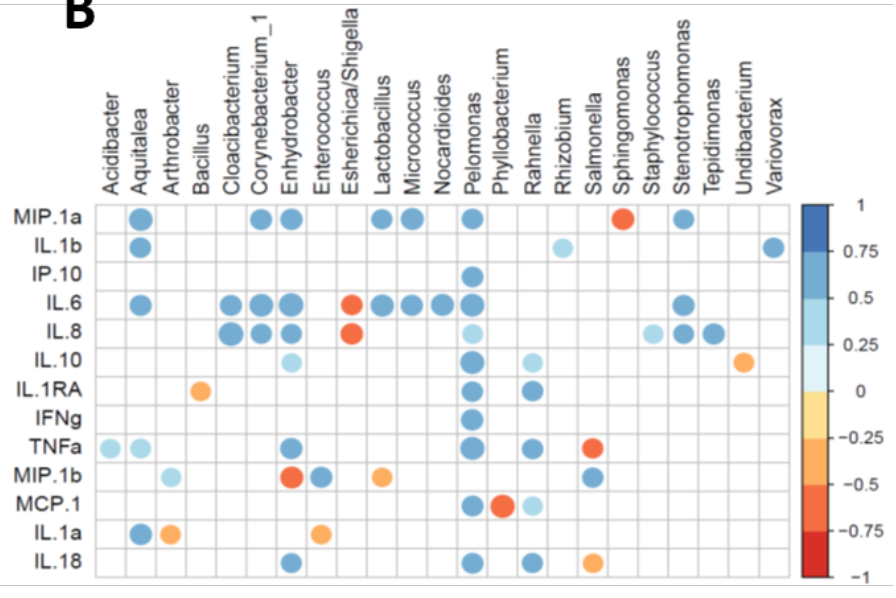

Figure 2. A) Cytokine levels were patient-specific and the clustering was driven by IP-10 (CXCL-10) and IL-1ra. Relative abundance of 65 identified genera in portal, hepatic and central venous, as well as peripheral blood. Heatmaps show relative abundance (scale showed on the right). Genera absent in a sample are marked by white boxes. B) Spearman correlations between microbial genus abundance and inflammatory markers. Only statistically significant correlations (adjusted $\mathrm{P}<0.05$ ) are shown. 


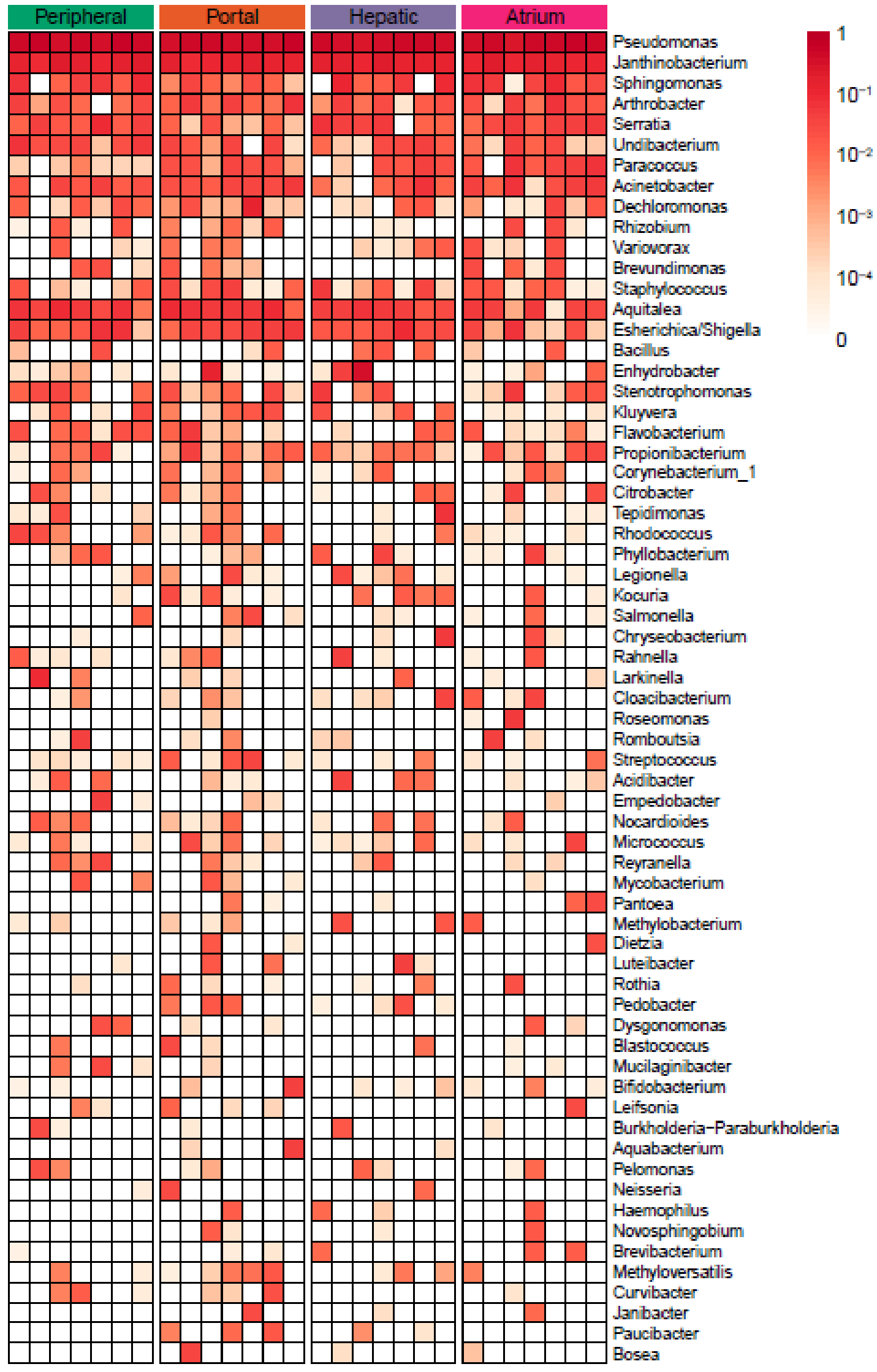

Supplemental Figure 1. Relative abundance of 65 identified genera in portal, hepatic and central venous, as well as peripheral blood from cohort A. Heatmaps show relative abundance (scale showed on the right). Genera absent in a sample are marked by white boxes. 
This article has been accepted for publication in Gut, 2018 following peer review, and the Version of Record can be accessed online at http://dx.doi.org/10.1136/gutjnl-2018-316227

(c) Article author(s) (or their employer(s) unless otherwise stated in the text of the article) 2019. 